\title{
Long non-coding RNA, LINC01614 as a potential biomarker for prognostic prediction in breast cancer
}

\author{
Yaozong Wang ${ }^{1}$, Baorong Song ${ }^{1}$, Zhu Leilei ${ }^{2}$, Xia Zhang ${ }^{\text {Corresp. } 3}$ \\ ${ }^{1}$ Department of General Surgery, HwaMei Hospital (Ningbo No.2 Hospital), University of Chinese Academy of Sciences, Ningbo, China \\ 2 Department of Radiotherapy, Shanghai East Hospital, Tongji University, Shanghai, China \\ 3 Breast Cancer Center, Shanghai East Hospital, Tongji University, Shanghai, China \\ Corresponding Author: Xia Zhang \\ Email address: zhangxia010203@126.com
}

Background Dysregulated IncRNAs may serve as potential biomarkers of cancers including breast cancer. This study aimed to identify IncRNAs with strong prognostic value for breast cancer.

Methods LncRNA expression profiles of 929 tissue samples were downloaded from TANRIC database. We performed differential expression analysis between paired breast cancer and adjacent normal tissues. Survival analysis was used to identify IncRNAs with prognostic value. Univariate and multivariate Cox regression analyses were performed to confirm the independent prognostic value of potential IncRNAs. Dysregulated signaling pathways associated with IncRNA expression were evaluated using GSEA.

Results We found that a total of 398 IncRNAs were significantly differentially expressed between breast cancer and adjacent normal tissues (adjusted $P$ value $<=0.0001$ and $|\log F C|>=1$ ). Additionally, 381 potential IncRNAs were correlated Overall Survival (P value $<0.05$ ). 48 IncRNAs remained when differentially expressed IncRNAs overlapped with IncRNAs that had prognostic value. Among the 48 IncRNAs, one IncRNA (LINC01614) had stronger prognostic value and was highly expressed in breast cancer tissues. LINC01614 expression was validated as an independent prognostic factor using univariate and multivariate analyses. Higher LINC01614 expression was observed in several molecular subgroups including ER+, PR+ and HER2 + subgroup, respectively. Also, breast cancer carrying one of four gene mutations had higher expression of LINC01614 including AOAH, CIT, HER2 and ODZ1. Higher expression of LINC01614 was positively correlated with several gene sets including TGF-beta1 response, CDH1 signals and cell adhesion pathways.

Conclusions A novel IncRNA LINC01614 was identified as a potential biomarker for prognosis prediction of breast cancer. This study emphasized the importance of LINC01614 and further research should be focused on it. 
1 Long non-coding RNA, LINC01614 as a potential biomarker for prognostic prediction in

2 breast cancer

3

4 Yaozong Wang ${ }^{1}$, Baorong Song ${ }^{1}$, Leilei Zhu², Xia Zhang ${ }^{3}$ I

5

$6{ }^{1}$ Department of General Surgery, HwaMei Hospital (Ningbo No.2 Hospital), University of Chinese

7 Academy of Sciences, Ningbo, China.

$8 \quad{ }^{2}$ Department of Radiotherapy, Shanghai East Hospital, Tongji University, Shanghai, China.

$9 \quad{ }^{3}$ Breast Cancer Center, Shanghai East Hospital, Tongji University, Shanghai, China

11 Corresponding author:

12 Xia Zhang

Breast Cancer Center, Shanghai East Hospital, Tongji University, Shanghai, China

Email address: zhangxia010203@126.com 


\section{Abstract}

Background Dysregulated IncRNAs may serve as potential biomarkers of cancers including breast cancer. This study aimed to identify IncRNAs with strong prognostic value for breast cancer.

Methods LncRNA expression profiles of 929 tissue samples were downloaded from TANRIC database. We performed differential expression analysis between paired breast cancer and adjacent normal tissues. Survival analysis was used to identify IncRNAs with prognostic value. Univariate and multivariate Cox regression analyses were performed to confirm the independent prognostic value of potential IncRNAs. Dysregulated signaling pathways associated with IncRNA expression were evaluated using GSEA.

Results We found that a total of 398 IncRNAs were significantly differentially expressed between breast cancer and adjacent normal tissues (adjusted $\mathrm{P}$ value $<=0.0001$ and $|\log F C|>=1$ ).

Additionally, 381 potential IncRNAs were correlated Overall Survival (P value < 0.05). 48 IncRNAs remained when differentially expressed IncRNAs overlapped with IncRNAs that had prognostic value. Among the 48 IncRNAs, one IncRNA (LINC01614) had stronger prognostic value and was highly expressed in breast cancer tissues. LINC01614 expression was validated as an independent prognostic factor using univariate and multivariate analyses. Higher LINC01614 expression was observed in several molecular subgroups including ER+, PR+ and HER2+ subgroup, respectively. Also, breast cancer carrying one of four gene mutations had higher expression of LINC01614 including AOAH, CIT, HER2 and ODZ1. Higher expression of LINC01614 was positively correlated with several gene sets including TGF-beta1 response, CDH1 signals and cell adhesion pathways. Conclusions A novel IncRNA LINC01614 was identified as a potential biomarker for prognosis 
prediction of breast cancer. This study emphasized the importance of LINC01614 and further research should be focused on it.

\section{Introduction}

Although the management of breast cancer (BRCA) has been significantly improved over the decades of years, it is still one of the most common cancers worldwide, especially for women. The morbidity and mortality of BRCA is continuously increasing in recent years especially in Europe countries such as the United States (Anderson et al. 2011). Based on the statistics from American Cancer Society, an estimated more than 260,000 BRCA cases occurred and more than 41,000 patients died because of BRCA in 2018 (Siegel et al. 2018). There are several common treatments for BRCA including surgical resection, chemotherapy, radiotherapy (De Vita 1989). Recently, immunotherapy is becoming a new strategy for some cancer types including BRCA (Hadden 1999). Since BRCA is a disease with high heterogeneity, the underlying mechanism of BRCA has not been totally elucidated (Garcia-Closas et al. 2008). Some BRCA cases were resistant to chemotherapy and these patients generally had worse survival. Recently, several molecules have been identified as biomarkers that can be used to determine response to therapy and prognostic prediction such as estrogen receptors (ERs), progesterone receptors (PRs) and (human epidermal growth factor receptor 2) HER2 (Colleoni \& Montagna 2012; Davies et al. 2011; Network 2012).

With the development of high throughput technique such as next generation sequencing, the biological function of long non-coding RNAs (IncRNAs) has been increasingly identified (Core et al. 2008). Indeed, more than $75 \%$ of the genome are transcribed into noncoding RNAs including 
IncRNAs and small RNAs such as tRNAs, rRNAs, miRNAs. Among these, IncRNAs are noncoding transcripts more than 200 nucleotide (nt) in length and are lack of protein-coding abilities (Huarte 2015). According to the Encyclopedia of DNA Elements (ENCODE) Project Consortium, more than

120,000 IncRNAs have been annotated (Jalali et al. 2016). Recently, dysregulated expression of IncRNAs have been found to be involved with cancer development and progression. Some critical IncRNAs have been identified as biomarkers and prognostic factors such as HOTAIR (Gupta et al. 2010), SAMMSON (Leucci et al. 2016), MALAT1 (Sun \& Ma 2019) and H19 (Zhang et al. 2016a).

Specifically, HOTAIR could increase cancer invasiveness and metastasis by reprograming chromatin state in a manner dependent on Polycomb repressive complex 2 (PRC2) (Gupta et al. 2010). LncRNA SAMMSON was found to interact with p32 and regulate homeostasis and metabolism of mitochondria. This effect could increase mitochondrial targeting and pro-oncogenic function of p32 (Leucci et al. 2016). Some other IncRNAs are identified to be involved with cell cycle control, tumor growth, drug response. Since IncRNAs may play important roles in the cancer carcinogenesis, it is necessary to identify potential IncRNAs that may serve as useful biomarkers and prognostic predictors by bioinformatic analysis.

This study was aimed to identify oncogenic IncRNAs with prognostic value in BRCA. LncRNAs were analyzed by correlating with tumorigenesis and Overall Survival (OS). 48 IncRNAs were differentially expressed between BRCA and adjacent normal tissues and also associated with OS. LncRNA

LINC01614 had higher expression in BRCA tissues and had stronger prognostic value than other candidate IncRNAs, thus, was selected for further analyses including correlation with clinical parameters, univariate and multivariate analyses using Cox regression model and identifying 
dysregulating signaling pathways using Gene Set Enrichment Analysis (GSEA).

\section{Materials and Methods}

\section{Clinical data collection}

LncRNA expression profile of BRCA was downloaded from TANRIC database

(https://ibl.mdanderson.org/tanric/_design/basic/index.html). All of these samples in TANRIC database were from the Cancer Genomic Atlas (TCGA, https://cancergenome.nih.gov/). A total of 929 tissue samples were collected and 105 of them were derived from paired normal tissues. The clinical information of these samples were downloaded from TCGA. 824 of these samples had survival information (OS). A total of 12727 IncRNAs were annotated in the expression profile. The clinical characteristics of these patients including age, gender, TNM stage, ER status, PR status, HER2 status, treatment history, PAM50 subtypes.

\section{Gene set enrichment analysis (GSEA)}

We used GSEA to identify dysregulated gene sets between high- and low-LINC01614 expression groups. GSEA was conducted by a JAVA program (http://software.broadinstitute.org/gsea/index.jsp) based on molecular signature database C2 CP (v6.2) which consisted of 4762 gene sets. The number of random sample permutations was set as 1000 . Generally, if the majority of members of a given gene set had higher expression accompanied with higher risk score, then gene set would have positive enrichment score and considered as enriched based on the nominal $P$ value $(<0.05)$, FDR $(<25 \%)$ and normalized enrichment score ( NES > 1) (Subramanian et al. 2005).

\section{Statistical analysis}


106 All of the statistical analyses were conducted by R (version 3.5.1). Differential expression analysis

107

108

109

110

111

112

113

114

115

116

117

118

119

120

121

122

123

124

125

126

between BRCA and adjacent normal tissues was performed using "Limma” R package (Ritchie et al.

2015). Two-sample t-test was used to test the statistical differences of LINC01614 expression

between two groups and ANOVA test for more than two groups (e.g., PAM50 subtypes). Chi-

squared test or Fisher's exact test was used to compare the clinical differences between high- and

low-LINC01614 expression groups. Kaplan-Meier estimate was used to measure the proportion of

patients living for a certain time period after surgery. Kaplan-Meier estimate was efficient to deal

with censored data. We visualized Kaplan-Meier estimate using "survival" R package. Survival

difference was compared using the log-rank test. Univariate and Multivariate Cox analysis were used

to test whether LINC01614 expression was an independent prognostic factor. Significance difference was confirmed as $\mathrm{P}$ value was less than 0.05 .

\section{Results}

\section{Identification of candidate IncRNAs with prognostic value in BRCA}

The work flow of this study was shown in Fig. S1. According to the TANRIC database, a total of

12727 IncRNAs were annotated. Firstly, we excluded IncRNAs that could not be detected in at least

$80 \%$ of these 824 samples. By doing so, 4537 IncRNAs remained for further analysis. Since paired

adjacent normal tissues in 105 of these samples were available, we performed differential

expression analysis between these 105 paired samples. By doing so, 398 IncRNAs were

differentially expressed between BRCA and adjacent normal tissues $(|\lg \mathrm{FC}|>=1$ and adjusted $\mathrm{P}$

value $<=0.0001$, Fig. 1A). To further screen out IncRNAs with prognostic value, we correlated these

4537 IncRNAs with OS. A total of 381 IncRNAs were found associated with OS (P value $<0.05$ and

Peer] reviewing PDF | (2019:05:38020:1:1:NEW 23 Aug 2019) 
$127|\mathrm{HR}|>1$, Table. S1). Subsequently, we identified 48 IncRNAs that were differentially expressed and

128 had prognostic value as well (Fig. 1B). Among six IncRNAs that were highly expressed in BRCA

129 tissues, one IncRNA named LINC01614 had stronger prognostic value than others $(\mathrm{HR}>1$ and $\mathrm{P}$

130 value $<0.01$,Fig. 1C, Table. S1). Thus, we selected LINC01614 for further analysis.

131 Correlation between LINC01614 expression and clinical characteristics in BRCA

132 The clinical characteristics of these 824 BRCA patients were shown in Table. S2. The age of these

133 patients ranged from 26 to 90 and the median was 58 . There were only 9 males in this cohort. Stage

134 I patients were found in 140 patients, stage II in 471, stage III in 178 and stage IV in 14 . Among

135 these patients, 573 were ER positive, 500 were PR positive and 110 were HER2 positive. Only 65

136 patients had treatment information and 41 of them were treated. In terms of PAM50 molecular

137 subtypes according to one paper in 2012 (Network 2012), 22 patients were classified as Normal-like

138 subtype, 138 patients were classified as Basal-like subtype, 64 patients were classified as HER2-

139 enriched subtype, 413 patients were classified as Luminal-A subtype and 187 patients were

140 classified as Luminal-B subtype.

141 These patients were classified into two groups based on the expression of LINC01614 and the

142 median expression was set as the cutoff value. The proportions of ER status $(p<0.0001), P R$

143 status $(p<0.0001)$, HER2 status $(p=0.0006)$ and PAM50 subtypes $(p<0.0001)$ were significantly

144 different between high- and low-expression group as shown in Table. S2. Although there was no

145 significant difference between these two groups $(P=0.076)$, there were more stage III and IV cases

146 in high-expression group $(n=102)$ than that in low-expression group $(n=90)$. We further compared the

147 expression differences among different molecular subgroups. Higher expression of LINC01614 was

Peer) reviewing PDF | (2019:05:38020:1:1:NEW 23 Aug 2019) 
148

149

150

151

152

153

154

155

156

157

158

159

160

161

162

163

164

165

166

167

168

observed in ER positive group( $P<0.0001$, Fig. $2 A$ ), PR positive group ( $P<0.0001$, Fig. $2 B$ ), and

HER2 positive group ( $P=0.0057$, Fig. 2C). Significant difference was also observed among PAM50

subtypes ( $P<0.0001$, Fig. 2D). Specifically, Luminal A and B had higher expression of LINC01614

followed by HER2, Normal and Basal subtypes.

Prognostic value of LINC01614 in BRCA

The median follow-up time was 32.5 months and only $15 \%$ of these patients were dead $(n=125)$.

Initially, we found that high-expression group had shorter survival than low-expression group ( $\mathrm{P}=$

$0.0076, \mathrm{HR}=1.63,95 \% \mathrm{Cl}=1.13-2.33, \mathrm{Fig} .3 \mathrm{~A})$. We also found that the prognostic value of

LINC01614 was also significant only in TNM stage II and III patients $(\mathrm{P}=0.01, \mathrm{HR}=1.71,95 \% \mathrm{Cl}=$

1.14-2.66, Fig. 3B). We included age, TNM stage, PR status, ER status, HER2 status and PAM50

subtypes for Univariate and Multivariate analysis using Cox regression model. Age was included as

a continuous variable in the model while the others were included as categorical variables. We did

not include gender in Cox regression model as there were only nine male patients and only one was

in low-expression group.

As for univariate Cox regression analysis, we found that high LINC01614 expression was

significantly associated with worse survival $(P=0.008, H R=1.63,95 \% C l=1.13-2.33$, Fig. $3 C)$ and

also for age $(P<0.0001, \mathrm{HR}=1.03,95 \% \mathrm{Cl}=1.02-1.05)$, stage III $(\mathrm{P}=0.001, \mathrm{HR}=2.72,95 \% \mathrm{Cl}=$

1.48-5.00), stage IV $(P<0.0001, H R=10.03,95 \% C I=4.58-21.90)$, PAM50 HER2 subtype $(P=$

0.007, $\mathrm{HR}=2.66,95 \% \mathrm{Cl}=1.31-5.39), \mathrm{PAM} 50$ Luminal $\mathrm{B}(\mathrm{P}=0.017, \mathrm{HR}=2.04,95 \% \mathrm{Cl}=1.14-$

3.67). We then performed multivariate Cox regression analysis and found that LINC01614

expression was an independent prognostic factor for BRCA $(P=0.007, \mathrm{HR}=1.88,95 \% \mathrm{Cl}=1.19$ -

Peer) reviewing PDF | (2019:05:38020:1:1:NEW 23 Aug 2019) 
169 2.97, Fig. 3D) as well as for age $(P<0.0001, H R=1.04,95 \% C l=1.02-1.06)$, stage III $(P=0.001$,

$170 \mathrm{HR}=3.72,95 \% \mathrm{Cl}=1.77-7.79)$, stage IV $(\mathrm{P}<0.0001, \mathrm{HR}=7.24,95 \% \mathrm{Cl}=2.94-17.86), \mathrm{PAM} 50$

171 HER2 subtypes $(P=0.021, H R=3.31,95 \% C l=1.20-9.13)$ and $H E R 2$ status $(P=0.026, H R=0.39$,

$17295 \% \mathrm{Cl}=0.17-0.89)$.

173 We further performed subgroup analyses based on PR status, ER status and HER2 status (Fig. S2).

174 Our results indicated that prognostic value of LINC01614 could be observed in ER positive group (P

$175=0.032, \mathrm{HR}=1.62,95 \% \mathrm{Cl}=1.04-2.53)$ and $\mathrm{HER} 2$ negative group $(\mathrm{P}=0.037, \mathrm{HR}=1.56,95 \% \mathrm{Cl}=$

176 1.02-2.37). Although there were no significant differences in other subgroups, we observed the trend that high-expression group had shorter survival than low-expression group among these subgroups.

178 More samples were needed to validate these results.

\section{Gene mutations correlated with LINC01614 expression}

We also explored the correlations between LINC01614 expression and gene mutations status using

181 the inbuilt functions of the TANRIC database. We found that the expression of LINC01614 was

182 significant associated with four gene mutations including AOHA, CIT, HER2 and ODZ1.

183 Consistently, patients carrying any one of these gene mutations had higher expression of

LINC01614 $(P=0.0064$ for $A O H A, P=0.0004$ for $C I T, P=0.0057$ for $H E R 2$ and $P=0.0011$ for

185 ODZ1, Fig. S3).

\section{Dysregulated pathways associated with LINC01614 expression}

We performed GSEA to identify dysregulated gene sets between high- and low-expression group.

799 and 42 gene sets were significantly (FDR $<25 \%$, normal $P$ value $<0.05)$ enriched in high- and 
190 in the development and progression of cancer such as TGF- $\beta 1$ response, CDHI signaling via

191 CTNNB1, integrin3 and integrin cell surface interaction pathways. High-expression group was also

192

193

194

195

196

197

198

199

200

201

202

203

204

205

206

207

208

209

210

enriched with one gene signature associated with ductal carcinoma. Finally, we also found that genes associated with Estradiol treatment were enriched in high-expression group (Fig. 4A-F).

\section{Discussion}

Taking the advantage of high-throughput sequencing technologies, increasingly comprehensive transcriptomes have been recognized and identified. The roles of IncRNAs have been investigated and emphasized (Huarte 2015). It has been found that IncRNAs play important roles in multiple biological processes including chromatin modification, transcription activation, transcription regulation (Yang et al. 2014). Accumulated studies have suggested that IncRNAs might be novel biomarkers for cancer diagnosis and prognosis prediction. The prognostic value of IncRNAs have been identified in several cancer types including colorectal cancer (Hu et al. 2014), non-small cell lung cancer (Zhou et al. 2015), gastric cancer (Zhu et al. 2016).

In this study, we made full use of comprehensive IncRNA expression profiles derived from TANRIC database. Among these IncRNAs, one IncRNA named LINC01614 (ENSG00000230838.1) had stronger prognostic value than others. High-expression group had shorter survival than that in lowexpression group. Further univariate and multivariate Cox regression analyses indicated that LINC01614 was an independent prognostic factor of BRCA. We further correlated the expression of LINC01614 with BRCA subtypes. Higher expression of LINC01614 was consistently observed in ER, PR, and HER2 positive group, respectively. Additionally, Luminal A, Luminal B and HER2 subgroups had relative higher expression of LINC01614 than that in Basal-like and Normal-like subgroups. The 
211 prognostic value of LINC01614 could be observed in ER positive and HER2 negative groups,

212 marginally in PR positive and negative subgroups, respectively. Some of the statistics were not

213 significant probably because of the small sample size of these subgroups. When correlated the

214 expression of LINC01614 with gene mutation status, we found the mutation status of four genes was

215 correlated with LINC01614 expression including AOAH, CIT, HER2 and ODZ1. Higher expression of

216 LINC01614 was observed in mutated samples. HER2 has been recognized as biomarker not only

217 just in BRCA (Chmielecki et al. 2015; Connell \& Doherty 2017). Most of HER2 mutation patients

218 responded to HER2 inhibitors (Ben-Baruch et al. 2015). AOHA was reported to have higher

219 conditional frequencies than marginal frequencies in early time intervals. This might indicate that

220 patients with AOAH mutation was more progressive (Sakoparnig et al. 2015). Citron kinase (CIT-K)

221 was also highly expressed in BRCA and could promote cell growth and colony formation, indicating

222 its oncogenic function in BRCA (Meng et al. 2018). Finally, ODZ1 was involved with increased cell

223 proliferation and invasion in glioblastoma (Talamillo et al. 2017) and was identified as a biomarker in

224 papillary thyroid carcinoma (Cheng et al. 2017).

225 Several critical pathways associated with TGF- $\beta 1$ response, CDHI signaling via CTNNB1, integrin3

226 and integrin cell surface interaction pathways were enriched in subgroup with high expression of

227 LINC01614. These dysregulated signaling pathways played important roles in the development and

228 progression of cancer (Liu \& Chu 2014; Seguin et al. 2015; Syed 2016). These results might imply

229 the potential function of LINC01614 in BRCA. In terms of the prognostic value, previous studies have

230 emphasized the prognostic value of LINC01614 in cancer. For instance, Huang et al identified three

231 IncRNAs with prognostic value in esophageal squamous cell carcinoma including RP11-366H4.1.1,

Peer) reviewing PDF | (2019:05:38020:1:1:NEW 23 Aug 2019) 
232 LINC00460 and LINC01614 (Huang et al. 2018). These three IncRNAs-based signature could

233 facilitate the prognosis prediction of esophageal squamous cell carcinoma. Moreover, Zhang et al

234 also found that LINC01614 was highly expression in ER positive BRCA which was in line with our

235 result (Zhang et al. 2016b). These findings highlighted the potential role of LINC01614 in BRCA and

236 it might be an useful biomarker for BRCA prognostic prediction.

237 There are several limitations for this study. Firstly, the prognostic value of LINC01614 should be

238 further validated in cohorts with larger sample size in the future. Secondly, the differential expression

239 of LINC01614 between BRCA and normal tissue should be validated through experiment. Thirdly,

240 the potential role of LINC01614 in BRCA should be experimentally explored and dysregulated

241 pathways identified in this study should also validated. Finally, as for the clinical practice, after the

242 potential role of LINC01614 is further confirmed, the clinicians can use it to facilitate prognosis

243 assessment of BRCA patients after surgery.

244 Conclusions: This research provided an example to identify candidate IncRNAs with prognostic

245 value in cancers. A novel IncRNA LINC01614 was identified as a potential biomarker for prognosis

246 prediction in BRCA. This study emphasized the importance of LINC01614 and further more research

247 should be focused on it.

Acknowledgments: The authors are grateful for all the subjects who participated in the study. Y. W.

250 and X. Z designed this research. Y. W., B. S. and L. Z. collected data and performed the analyses.

251 Y. W. drafted the manuscript. Y. W., B. S., L. Z. and X. Z read and revised the manuscript.

252 Funding: This work was supported by grants from Shanghai Pudong New Area Health and Family 
253

254

255

256

257

258

259

260

261

262

263

264

265

266

267

268

269

270

271

272

273

274

275

276

277

278

279

280

281

282

283

284

285

286

287

288

289

Planning Commission Health Science and Technology Project, NO\# PW2016B-5.

Competing Interests: The authors declare that there are no conflicts of interest.

\section{References:}

Anderson WF, Katki HA, and Rosenberg PS. 2011. Incidence of breast cancer in the United States: current and future trends. J Natl Cancer Inst 103:1397-1402. 10.1093/jnci/djr257

Ben-Baruch NE, Bose R, Kavuri SM, Ma CX, and Ellis MJ. 2015. HER2-Mutated Breast Cancer Responds to Treatment With Single-Agent Neratinib, a Second-Generation HER2/EGFR Tyrosine Kinase Inhibitor. $J$ Natl Compr Canc Netw 13:1061-1064.

Cheng SP, Chen MJ, Chien MN, Lin CH, Lee JJ, and Liu CL. 2017. Overexpression of teneurin transmembrane protein 1 is a potential marker of disease progression in papillary thyroid carcinoma. Clin Exp Med 17:555564. 10.1007/s10238-016-0445-y

Chmielecki J, Ross JS, Wang K, Frampton GM, Palmer GA, Ali SM, Palma N, Morosini D, Miller VA, Yelensky R, Lipson D, and Stephens PJ. 2015. Oncogenic alterations in ERBB2/HER2 represent potential therapeutic targets across tumors from diverse anatomic sites of origin. Oncologist 20:7-12. 10.1634/theoncologist.20140234

Colleoni M, and Montagna E. 2012. Neoadjuvant therapy for ER-positive breast cancers. Ann Oncol 23 Suppl 10:x243-248. 10.1093/annonc/mds305

Connell CM, and Doherty GJ. 2017. Activating HER2 mutations as emerging targets in multiple solid cancers. ESMO Open 2:e000279. 10.1136/esmoopen-2017-000279

Core LJ, Waterfall JJ, and Lis JT. 2008. Nascent RNA sequencing reveals widespread pausing and divergent initiation at human promoters. Science 322:1845-1848. 10.1126/science.1162228

Davies C, Godwin J, Gray R, Clarke M, Cutter D, Darby S, McGale P, Pan HC, Taylor C, Wang YC, Dowsett M, Ingle J, and Peto R. 2011. Relevance of breast cancer hormone receptors and other factors to the efficacy of adjuvant tamoxifen: patient-level meta-analysis of randomised trials. Lancet 378:771-784. 10.1016/s01406736(11)60993-8

De Vita VT, Jr. 1989. Breast cancer therapy: exercising all our options. $N$ Engl J Med 320:527-529. 10.1056/nejm198902233200812

Garcia-Closas M, Hall P, Nevanlinna H, Pooley K, Morrison J, Richesson DA, Bojesen SE, Nordestgaard BG, Axelsson CK, Arias JI, Milne RL, Ribas G, Gonzalez-Neira A, Benitez J, Zamora P, Brauch H, Justenhoven C, Hamann U, Ko YD, Bruening T, Haas S, Dork T, Schurmann P, Hillemanns P, Bogdanova N, Bremer M, Karstens JH, Fagerholm R, Aaltonen K, Aittomaki K, von Smitten K, Blomqvist C, Mannermaa A, Uusitupa M, Eskelinen M, Tengstrom M, Kosma VM, Kataja V, Chenevix-Trench G, Spurdle AB, Beesley J, Chen X, Devilee P, van Asperen CJ, Jacobi CE, Tollenaar RA, Huijts PE, Klijn JG, Chang-Claude J, Kropp S, Slanger T, Flesch-Janys D, Mutschelknauss E, Salazar R, Wang-Gohrke S, Couch F, Goode EL, Olson JE, Vachon C, Fredericksen ZS, Giles GG, Baglietto L, Severi G, Hopper JL, English DR, Southey MC, Haiman CA, Henderson BE, Kolonel LN, Le Marchand L, Stram DO, Hunter DJ, Hankinson SE, Cox DG, Tamimi R, 
Kraft P, Sherman ME, Chanock SJ, Lissowska J, Brinton LA, Peplonska B, Klijn JG, Hooning MJ, MeijersHeijboer H, Collee JM, van den Ouweland A, Uitterlinden AG, Liu J, Lin LY, Yuqing L, Humphreys K, Czene K, Cox A, Balasubramanian SP, Cross SS, Reed MW, Blows F, Driver K, Dunning A, Tyrer J, Ponder BA, Sangrajrang S, Brennan P, McKay J, Odefrey F, Gabrieau V, Sigurdson A, Doody M, Struewing JP, Alexander B, Easton DF, and Pharoah PD. 2008. Heterogeneity of breast cancer associations with five susceptibility loci by clinical and pathological characteristics. PLoS Genet 4:e1000054. 10.1371/journal.pgen.1000054

Gupta RA, Shah N, Wang KC, Kim J, Horlings HM, Wong DJ, Tsai MC, Hung T, Argani P, Rinn JL, Wang Y, Brzoska P, Kong B, Li R, West RB, van de Vijver MJ, Sukumar S, and Chang HY. 2010. Long non-coding RNA HOTAIR reprograms chromatin state to promote cancer metastasis. Nature 464:1071-1076. 10.1038/nature08975

Hadden JW. 1999. The immunology and immunotherapy of breast cancer: an update. Int J Immunopharmacol 21:79101.

Hu Y, Chen HY, Yu CY, Xu J, Wang JL, Qian J, Zhang X, and Fang JY. 2014. A long non-coding RNA signature to improve prognosis prediction of colorectal cancer. Oncotarget 5:2230-2242. 10.18632/oncotarget. 1895

Huang GW, Xue YJ, Wu ZY, Xu XE, Wu JY, Cao HH, Zhu Y, He JZ, Li CQ, Li EM, and Xu LY. 2018. A threelncRNA signature predicts overall survival and disease-free survival in patients with esophageal squamous cell carcinoma. BMC Cancer 18:147. 10.1186/s12885-018-4058-6

Huarte M. 2015. The emerging role of IncRNAs in cancer. Nat Med 21:1253-1261. 10.1038/nm.3981

Jalali S, Gandhi S, and Scaria V. 2016. Navigating the dynamic landscape of long noncoding RNA and protein-coding gene annotations in GENCODE. Hum Genomics 10:35. 10.1186/s40246-016-0090-2

Leucci E, Vendramin R, Spinazzi M, Laurette P, Fiers M, Wouters J, Radaelli E, Eyckerman S, Leonelli C, Vanderheyden K, Rogiers A, Hermans E, Baatsen P, Aerts S, Amant F, Van Aelst S, van den Oord J, de Strooper B, Davidson I, Lafontaine DL, Gevaert K, Vandesompele J, Mestdagh P, and Marine JC. 2016. Melanoma addiction to the long non-coding RNA SAMMSON. Nature 531:518-522. 10.1038/nature17161

Liu X, and Chu KM. 2014. E-cadherin and gastric cancer: cause, consequence, and applications. Biomed Res Int 2014:637308. 10.1155/2014/637308

Meng D, Yu Q, Feng L, Luo M, Shao S, Huang S, Wang G, Jing X, Tong Z, Zhao X, and Liu R. 2018. Citron kinase (CIT-K) promotes aggressiveness and tumorigenesis of breast cancer cells in vitro and in vivo: preliminary study of the underlying mechanism. Clin Transl Oncol. 10.1007/s12094-018-02003-9

Network CGA. 2012. Comprehensive molecular portraits of human breast tumours. Nature 490:61-70. 10.1038/nature11412

Ritchie ME, Phipson B, Wu D, Hu Y, Law CW, Shi W, and Smyth GK. 2015. limma powers differential expression analyses for RNA-sequencing and microarray studies. Nucleic Acids Res 43:e47. 10.1093/nar/gkv007

Sakoparnig T, Fried P, and Beerenwinkel N. 2015. Identification of constrained cancer driver genes based on mutation timing. PLoS Comput Biol 11:e1004027. 10.1371/journal.pcbi.1004027

Seguin L, Desgrosellier JS, Weis SM, and Cheresh DA. 2015. Integrins and cancer: regulators of cancer stemness, metastasis, and drug resistance. Trends Cell Biol 25:234-240. 10.1016/j.tcb.2014.12.006

Siegel RL, Miller KD, and Jemal A. 2018. Cancer statistics, 2018. CA: A Cancer Journal for Clinicians 68:7-30. $10.3322 /$ caac. 21442

Subramanian A, Tamayo P, Mootha VK, Mukherjee S, Ebert BL, Gillette MA, Paulovich A, Pomeroy SL, Golub TR, 
331

332

333

334

335

336

337

338

339

340

341

342

343

344

345

346

347

348

349

350

351

352

353
Lander ES, and Mesirov JP. 2005. Gene set enrichment analysis: a knowledge-based approach for interpreting genome-wide expression profiles. Proc Natl Acad Sci U S A 102:15545-15550. 10.1073/pnas.0506580102

Sun Y, and Ma L. 2019. New Insights into Long Non-Coding RNA MALAT1 in Cancer and Metastasis. Cancers (Basel) 11. 10.3390/cancers 11020216

Syed V. 2016. TGF-beta Signaling in Cancer. J Cell Biochem 117:1279-1287. 10.1002/jcb.25496

Talamillo A, Grande L, Ruiz-Ontanon P, Velasquez C, Mollinedo P, Torices S, Sanchez-Gomez P, Aznar A, EsparisOgando A, Lopez-Lopez C, Lafita C, Berciano MT, Montero JA, Vazquez-Barquero A, Segura V, Villagra NT, Pandiella A, Lafarga M, Leon J, Martinez-Climent JA, Sanz-Moreno V, and Fernandez-Luna JL. 2017. ODZ1 allows glioblastoma to sustain invasiveness through a Myc-dependent transcriptional upregulation of RhoA. Oncogene 36:1733-1744. 10.1038/onc.2016.341

Yang L, Froberg JE, and Lee JT. 2014. Long noncoding RNAs: fresh perspectives into the RNA world. Trends Biochem Sci 39:35-43. 10.1016/j.tibs.2013.10.002

Zhang K, Luo Z, Zhang Y, Zhang L, Wu L, Liu L, Yang J, Song X, and Liu J. 2016a. Circulating lncRNA H19 in plasma as a novel biomarker for breast cancer. Cancer Biomark 17:187-194. 10.3233/cbm-160630

Zhang Y, Wagner EK, Guo X, May I, Cai Q, Zheng W, He C, and Long J. 2016b. Long intergenic non-coding RNA expression signature in human breast cancer. Sci Rep 6:37821. 10.1038/srep37821

Zhou M, Guo M, He D, Wang X, Cui Y, Yang H, Hao D, and Sun J. 2015. A potential signature of eight long noncoding RNAs predicts survival in patients with non-small cell lung cancer. J Transl Med 13:231. 10.1186/s12967-015-0556-3

Zhu X, Tian X, Yu C, Shen C, Yan T, Hong J, Wang Z, Fang JY, and Chen H. 2016. A long non-coding RNA signature to improve prognosis prediction of gastric cancer. Mol Cancer 15:60. 10.1186/s12943-016-0544-0 
Figure 1

Fig. 1. Expression profiles of candidate IncRNAs.

Fig. 1. Expression profiles of candidate IncRNAs. (A) Heatmap of 398 IncRNA expression profiles that were differentially expressed between BRCA and adjacent normal tissues (adjusted $\mathrm{P}$ value $<=0.0001,|\log \mathrm{FC}|>=1$ ). Rows represent IncRNAs, and columns represent patients. (B) Heatmap of 48 IncRNA expression profiles that were differentially expressed in (A) with prognostic value ( $P$ value $<0.05$ ). (C) Boxplot of candidate IncRNA (LINC01614) between BRCA and paired normal tissues.
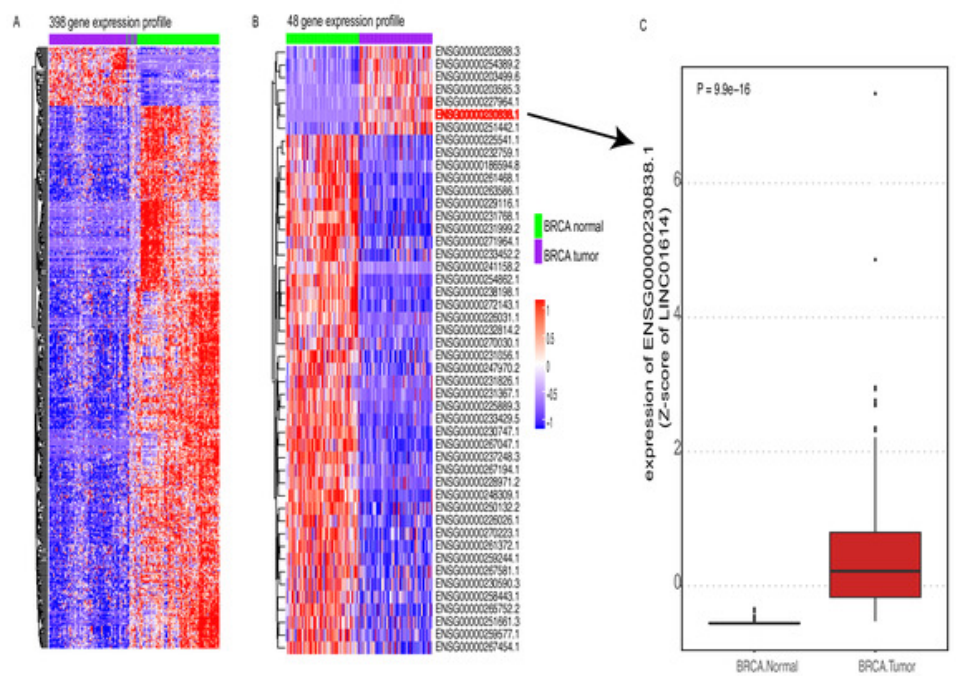
Figure 2

Fig. 2. Expression comparison of LINC01614 among molecular subgroups or subtypes of BRCA.

Fig. 2. Expression comparison of LINC01614 among molecular subgroups or subtypes of BRCA. (A) ER positive versus ER negative. (B) PR positive versus PR negative. (C) HER2 positive versus HER2 negative. (D) PAM50 molecular subtypes.
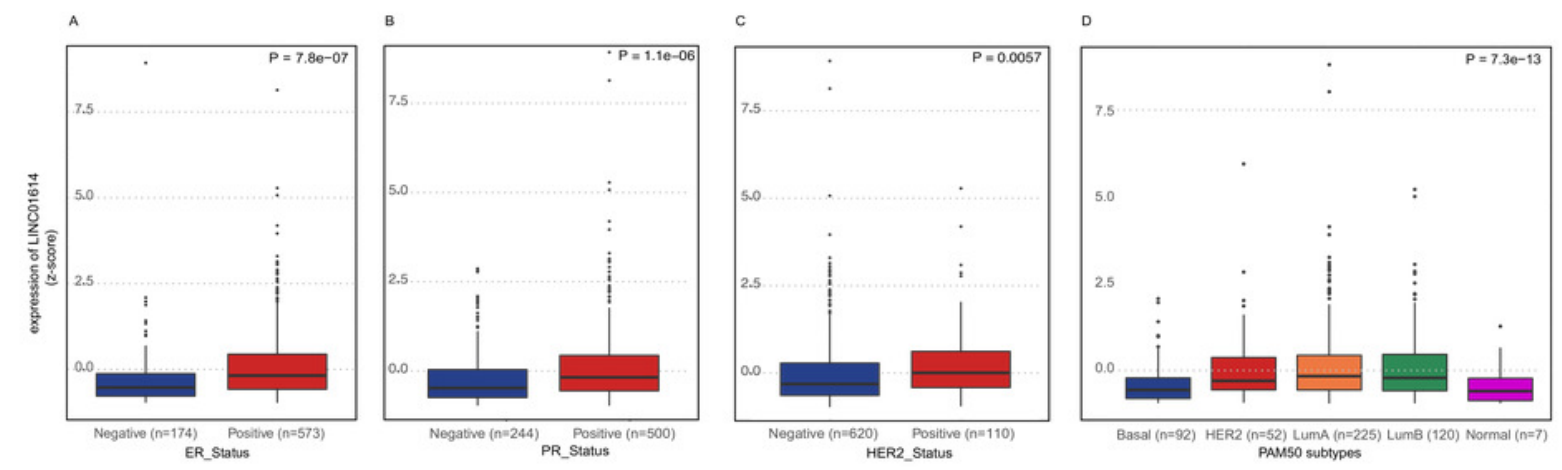


\section{Figure 3}

Fig. 3. Survival analysis of LINC01614 based on two groups classified using the median expression of LINC01614.

Fig. 3. Survival analysis of LINC01614 based on two groups classified using the median expression of LINC01614. (A) Kaplan-Meier estimates of Overall Survival in 824 BRCA samples. (B) Kaplan-Meier estimates of Overall Survival stage II and III BRCA samples ( $\mathrm{n}=$ 647). The tick marks on the Kaplan-Meier curves indicated the censored cases. The differences between the two curves were determined by the two-side log-rank test. (C) Univariate Cox regression proportional hazards regression analysis between clinical features. (D) Multivariate Cox regression proportional hazards regression analysis of LINC0614 expression groups adjusted by other clinical features including age, TNM stage, PAM50, ER, PR and HER2 status. Age was regarded as continuous variable. Gender was not included because there were only nine male subjects and only one in low-expression group. Solid squares indicated the hazard ratio (HR) of death, and close-ended horizontal lines represent the $95 \%$ confidence intervals $(\mathrm{Cl})$. All P values were calculated using Cox regression hazards analysis. 


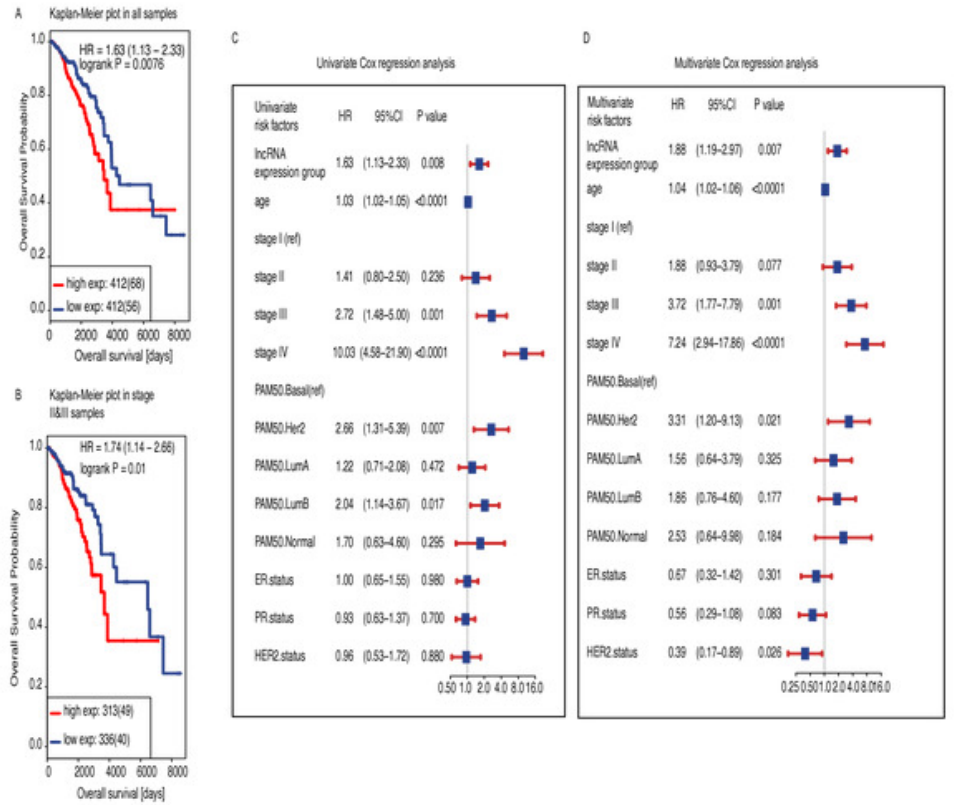


Figure 4

Fig. 4. Enrichment plots of GSEA based on LINC01614 expression group.

Fig. 4. Enrichment plots of GSEA based on LINC01614 expression group. GSEA indicated that TGF-beta1 response (A), CDHI signaling via CTNNB1 (B), integrin3 (C) and integrin cell surface interaction (D), ductal carcinoma signature $(E)$ and Estradiol treatment response signature were significantly enriched in high-expression group. NES, normalized ES; FDR: false discovery rate. 
A

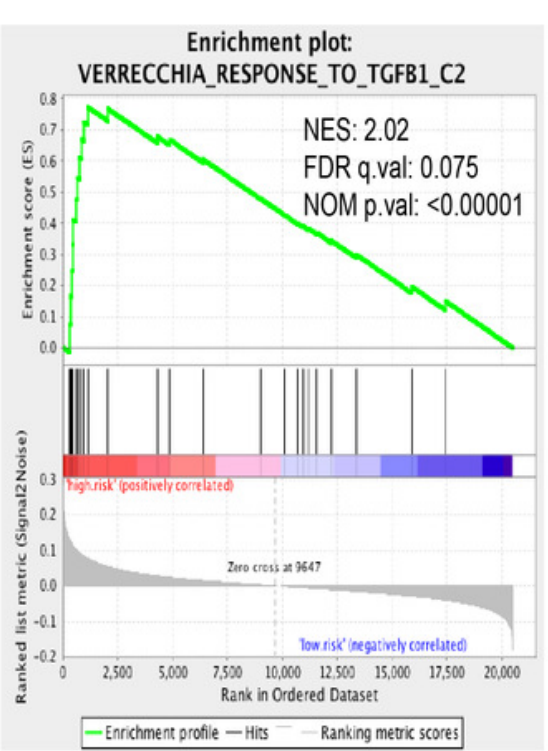

D

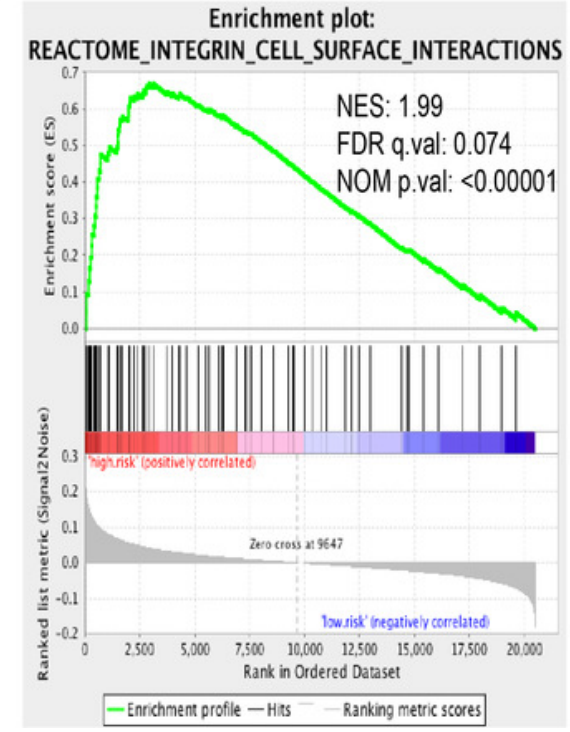

B

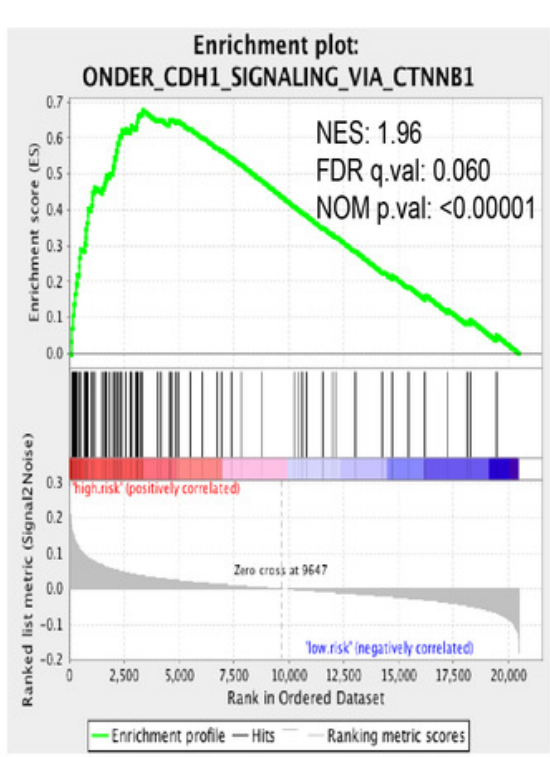

E

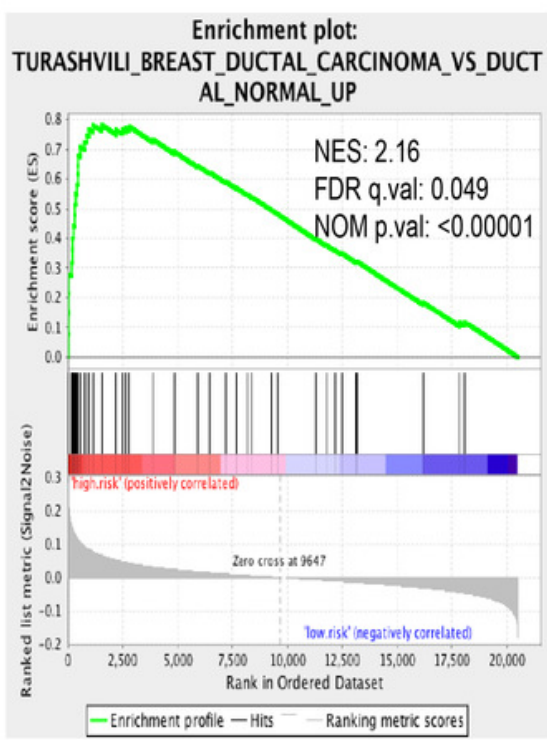

C

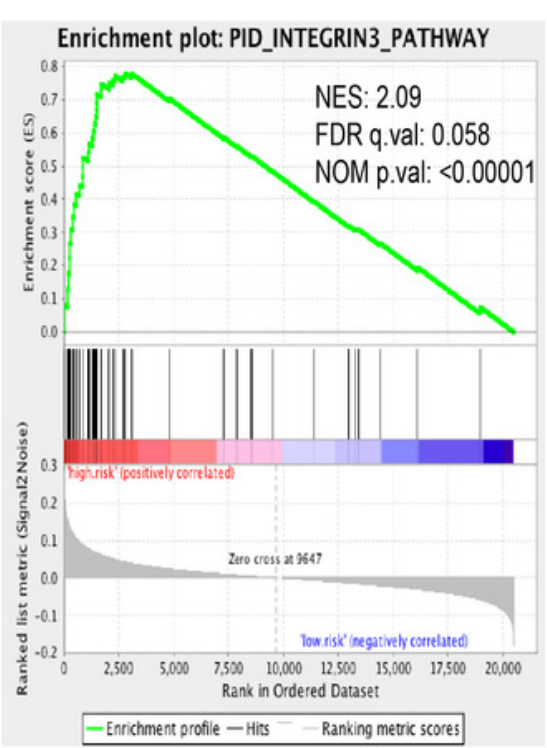

F

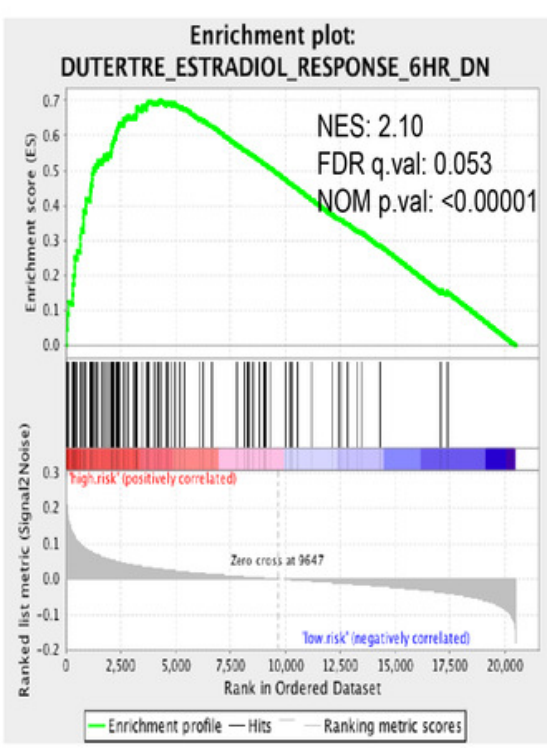


Figure 5

Fig. 2. Expression comparison of LINC01614 among molecular subgroups or subtypes of BRCA.

Fig. 2. Expression comparison of LINC01614 among molecular subgroups or subtypes of BRCA. (A) ER positive versus ER negative. (B) PR positive versus PR negative. (C) HER2 positive versus HER2 negative. (D) PAM50 molecular subtypes.
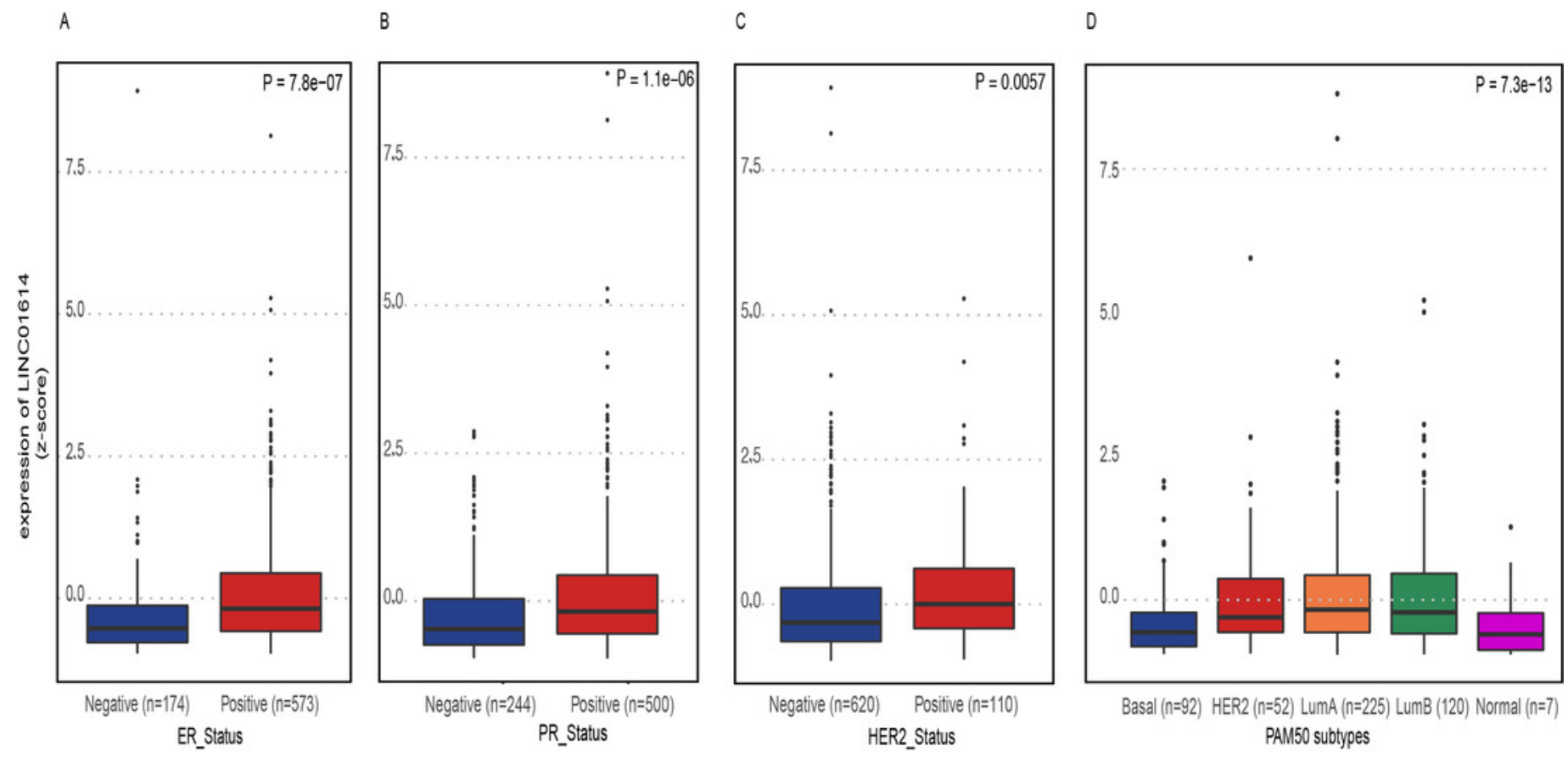